\title{
Three general concepts to improve risk prediction: good data, wisdom of the crowd, recalibration [version 1; peer review: 2
}

\section{approved with reservations]}

\author{
Ivan Kondofersky ${ }^{1,2}$, Michael Laimighofer ${ }^{1,2}$, Christoph Kurz $^{3}$, \\ Norbert Krautenbacher 1,2, Julia F. Söllner1, Philip Dargatz ${ }^{4}$, Hagen Scherb1, \\ Donna P. Ankerst 2,5 , Christiane Fuchs (D1,2
}

\footnotetext{
${ }^{1}$ Institute of Computational Biology, Helmholtz Zentrum München, German Research Center for Environmental Health, Neuherberg, Germany

${ }^{2}$ Mathematical Modeling of Biological Systems, Center for Mathematics, Technical University of Munich, Garching, Germany ${ }^{3}$ Institute of Health Economics and Health Care Management, Helmholtz Zentrum München, German Research Center for Environmental Health, Neuherberg, Germany

${ }^{4}$ Department of Hematology and Oncology, Johannes Wesling Klinikum Minden, Minden, Germany

5 University of Texas Health Science Center at San Antonio, San Antonio, USA
}

V1 First published: 16 Nov 2016, 5:2671
https://doi.org/10.12688/f1000research.8680.1

Latest published: 16 Nov 2016, 5:2671

https://doi.org/10.12688/f1000research.8680.1

\section{Open Peer Review}

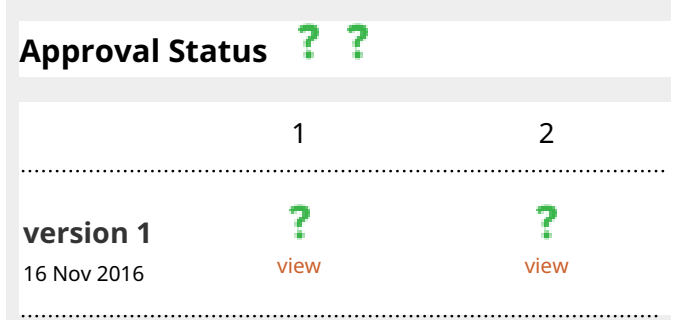

1. Sebastian Pölsterl ID, Institute for Cancer Research, London, UK

2. Riccardo de Bin, University of Oslo, Oslo, Norway

Any reports and responses or comments on the article can be found at the end of the article. 


\section{Keywords}

prostate cancer, survival prediction, crowdsourcing , community

challenge, DREAM, recalibration, validation, model averaging

\footnotetext{
Corresponding author: Christiane Fuchs (christiane.fuchs@helmholtz-muenchen.de)

Competing interests: No competing interests were disclosed.
}

Grant information: CF and IK are supported by the European Union within the ERC grant LatentCauses. CF is supported by the German Research Foundation (DFG) within the Collaborative Research Centre 1243, Subproject A17.

The funders had no role in study design, data collection and analysis, decision to publish, or preparation of the manuscript.

Copyright: (c) 2016 Kondofersky I et al. This is an open access article distributed under the terms of the Creative Commons Attribution License, which permits unrestricted use, distribution, and reproduction in any medium, provided the original work is properly cited.

How to cite this article: Kondofersky I, Laimighofer M, Kurz C et al. Three general concepts to improve risk prediction: good data, wisdom of the crowd, recalibration [version 1; peer review: 2 approved with reservations] F1000Research 2016, 5:2671 https://doi.org/10.12688/f1000research.8680.1

First published: 16 Nov 2016, 5:2671 https://doi.org/10.12688/f1000research.8680.1 


\section{Introduction}

Government funded clinical and research trials are currently experiencing increased pressure to publish comprehensive anonymized data in order to maximize scientific output, ushering in new challenges and opportunities for data scientists ${ }^{1}$. In an era of personalized medicine, scientists analyzing the results of large population-based clinical and prevention trials are further encouraged to translate results to clinical practice. With patient as the consumer, this push has led to an explosion of easy-to-use online clinical risk prediction tools for nearly all types of clinical outcomes $^{2,3}$. In the past, single-study prediction models dominated out of convenience. In the current climate, multiple studies are available that can be combined, increasing accuracy through the wisdom-of-the-crowd philosophy, and providing more realistic estimates of variability for decision-making. Ensembles or collections of models have been shown to outperform top-nominated models ${ }^{4}$.

Following efforts by Project Data Sphere to coordinate the release of comparative arm data from multiple pharmaceutical companies and academic medical centers, and in cooperation with the Dialogue for Reverse Engineering Assessments and Methods (DREAM) initiative, the Prostate Cancer DREAM Challenge sought to facilitate the development of survival prediction models to assist patients with metastatic castration-resistant prostate cancer (mCRPC) treated with first-line docetaxel ${ }^{5,6}$. Baseline and follow-up data were available from 1600 patients who had received first-line docetaxel as part of their participation on the comparator arms of three clinical trials, which formed the training set; see Guinney et al. ${ }^{7}$ and https://www. synapse.org/ProstateCancerChallenge for a detailed description. This article focuses on the challenge of predicting overall patient survival (sub-challenge 1). Here, data from 157 patients from an independent trial were made available for calibration to the target, and the final model based on the training and calibration data was validated on 313 patients from the target. An open online competition format with multiple deadlines attracted researchers from around the world, encouraging efficiency and fast-paced targeted research towards a common goal of optimizing predictive accuracy of a tool on an external test set.

There is no uniform prescription for building a universally optimal risk prediction tool. In the past, researchers often focused on a small set of standard risk factors for data cleaning and inclusion in their models, either for statistical reasons or grounds content; see Kattan et al. ${ }^{8}$ for the American Joint Committee on Cancer (AJCC)'s criteria for a prognostic model. The ever more commonly performed indiscriminate data-dumps from multiple clinical trials bring forth additional challenges of signal discovery, data cleaning, and missing data adjustment. Today's data scientist has to decide which datasets to use for training the models versus which to hold out for testing, as well as how to use initial information from the target population to fine-tune the model. The Prostate Cancer DREAM Challenge provided participating teams with hands-on experience in these critical areas. Through our participation in the challenge, we experimented with hundreds of models, data inclusion and missing-value adjustment options. By the end of the process, three general principles stood out that proved crucial to success: good data, wisdom of the crowds, and recalibration. Herein, we illustrate these principles and quantify their impact.

\section{Preliminaries}

The goal of sub-challenge 1 was to develop a survival prediction model using data from three different clinical trials, which was to be validated on data from a fourth independent trial. Random subsets of data from the fourth validation trial were provided at multiple interim points to guide model construction. After trying several machine learning and statistical models, the combined Cox proportional hazards and lasso model was chosen as it performed optimally on the interim validation sets ${ }^{9}$. The Cox proportional hazards model specifies the mortality hazard rate for an individual with covariate vector $x$ as:

$$
\lambda(t \mid x)=\lambda_{0}(\mathrm{t}) \exp \left(x^{\prime} \beta\right),
$$

where $\beta$ is the vector of $\log$ hazard ratios for respective covariates comprising $x$, and $\lambda_{0}(t)$ is a baseline hazard function that is left unspecified, making the model semi-parametric and more flexible than fully-specified parametric survival models. The model follows proportional hazards since the ratio of hazards for an individual with a unit increase in a single covariate relative to another individual, with all other covariates fixed, equals $\exp (\beta)$, which is constant for all times $t$. The non-parametric Kaplan-Meier estimator shows the empirical distribution of the observed failure times subject to censoring. Inspection of whether the curves stratified by different covariate values remain separated across the length of follow-up can be used to informally assess whether the proportional assumption holds.

The standard method for estimating $\beta$ in the Cox model is based on the partial likelihood that specifies for each individual their relative probability of failure compared to other individuals at risk:

$$
L(\beta)=\prod_{r \in D} \frac{\exp \left(\beta^{\prime} x_{r}\right)}{\sum_{j \in R_{r}} \exp \left(\beta^{\prime} x_{j}\right)} .
$$

In this formulation, $D$ is the group of distinct death times observed in the study, and $R_{j}$ denotes the risk set of all individuals still alive and on-study. If multiple individuals have the same death time, modifications are needed for the likelihood, which are implemented using a choice of algorithms.

Instead of finding the $\beta$ that maximizes the $\log$ likelihood $\ell(\beta)=\log L(\beta)$ itself, the lasso (least absolute shrinkage and selection operator) maximizes it subject to the constraint that $\Sigma_{j}\left|\beta_{j}\right|<s$, where $s$ is a user-selected tuning parameter. This modification heuristically keeps model dimensionality low, with unnecessary parameters shrunk to zero as necessary.

Sub-challenge 1 was again divided into two tasks: In sub-challenge 1a, participants were asked to predict risks of death. Sub-challenge $1 \mathrm{~b}$ asked for the prediction of exact times until death. For evaluating the proposed prediction models on the withheld test data, two criteria were used, corresponding to sub-challenges $1 \mathrm{a}$ and $1 \mathrm{~b}$, respectively. The evaluation criterion for 1 a focused on discrimination, that is how well the risk prediction model differentiated a patient about to experience mortality versus not. This criterion only compared the ranks of risk scores among groups of patients, with no 
further regard to accuracy in terms of actual values of risk scores. The second criterion of calibration focused on accuracy in terms of how close the exact time to event (death) was to the predicted time to event.

Receiver-operator-characteristics (ROC) curves have their origin in radar technology and signal processing and remain the standard of choice for determining the discrimination capability of a diagnostic test ${ }^{10}$. They have been most widely used for evaluating prediction models for binary disease outcomes based on retrospective case-control studies. In this context, the idea is that risk prediction tools return a probability between 0 and 1 of an individual having a disease, and any value, say $c$, could be used as a threshold for making a yes/no decision concerning whether the person is diseased, warranting further diagnostic work-up. A person with predicted risk exceeding $c$ is labeled as testing positive for disease and a person with risk less than or equal to $c$ as negative. Given a set of diseased cases and non-diseased controls, each with a predicted risk $p_{r}$, for every threshold $c$ there exist two measures of correct prediction, one for the cases and one for the controls, respectively:

$$
\begin{gathered}
\operatorname{Sensitivity}(c)=P\left(p_{r}>c \mid \text { Diseased }\right), \\
\text { Specificity }(c)=P\left(p_{r} \leq c \mid \text { Not Diseased }\right),
\end{gathered}
$$

The ROC curve displays the sensitivity, also termed the true positive rate, against 1-specificity, also termed the false positive rate for all possible choices of $c$. The area under the ROC curve (AUC) can therefore be used as a metric for model evaluation and comparison. It may be interpreted as a concordance index, where a value of 1 $(100 \%)$ represents perfect accuracy (i.e. sensitivity and specificity of 1 ) and a value of 0.5 equals random guessing.

For extension to prediction of survival up until fixed time periods that accommodate censored observations, Heagerty et al. ${ }^{11}$ proposed time-dependent ROC curves using time-specific versions of sensitivity and specificity that were based on whether individuals still on study were alive (controls) versus not (cases) at each time $t$, yielding as a result a plot of AUC values versus time $t$. Hung et $a l .{ }^{12}$ provided non-parametric estimators for the timedependent AUC and Blanche et al. ${ }^{13}$ provided an $\mathrm{R}$ package timeROC that was used for evaluation in sub-challenge 1a. To arrive at a single measure, integrated AUCs from 6 to 30 months were calculated and referred to as iAUCs.

Calibration measures the accuracy of numerical predictions, answering the question of how close estimates are to the truth. For sub-challenge $1 \mathrm{~b}$, which aimed at predicting the time to event (actual day of mortality), the root mean squared error (RMSE) was used:

$$
\mathrm{RMSE}=\sqrt{\frac{1}{\sum_{i=1}^{n} D_{i}} \sum_{i=1}^{n} D_{i}\left(\hat{y}_{i}-y_{i}\right)^{2}},
$$

where $\hat{y}$ is a vector of $n$ predictions for all patients in the test set, $y$ is the vector of $n$ observed values (which equals NA in case death is not observed), $D_{i}$ is a binary variable equal to one if death is reported and zero otherwise, and subscripts denote individual predictions and observed values on the test set. Thus, the RMSE was only calculated on patients with observed death times on study, and $\sum_{i=1}^{n} D_{i}$ referred to the number of death event times in the test set.

\section{Methods}

First concept: good data

Figure 1 gives an overview of the Prostate Cancer DREAM Challenge data after some cleaning (see low-cost strategy in paragraph below) but before inclusion of additional variables. There were six data tables available: one core table (the basis of Figure 1), containing baseline clinical covariates at patient level, and five longitudinal data tables, containing additional information at event level. We refer to the four trials as ASCENT-2 (Novacea, provided by Memorial Sloan Kettering Cancer Center ${ }^{14}$ ), VENICE (Sanofi ${ }^{15}$ ), MAINSAIL $\left(\right.$ Celgene $^{16}$ ), and ENTHUSE-33 (AstraZeneca ${ }^{17}$ ). The majority of the variables $(73.95 \%)$ in the core table have been measured in all four studies. Eight variables (albumin, magnesium, sodium, total protein, phosphorus, region and presence of target and non-target lesions) were exclusive to MAINSAIL, ENTHUSE-33 and VENICE while two (red blood cells and lymphocytes) were only assessed in ENTHUSE-33 and MAINSAIL. Lactate dehydrogenase was only measured in ASCENT-2, ENTHUSE-33 and MAINSAIL but not in VENICE. The presence of neoplasms and creatinine clearance were only present in VENICE and ENTHUSE-33. Unfortunately, the interesting variable gleason score was only reported in the ASCENT-2 study. With a p-value of 0.0017 it proved to be highly significant in a univariate Cox model for those patients where the variable was available, but was removed by us due to its missingness in the test dataset ENTHUSE-33. The significance of other variables which were missing in at least one trial is presented in Table 1.

In this section we compare two strategies to secure as many data elements as possible: a relatively straightforward low-cost minimal adaptation approach versus a high-cost strategy that incorporates

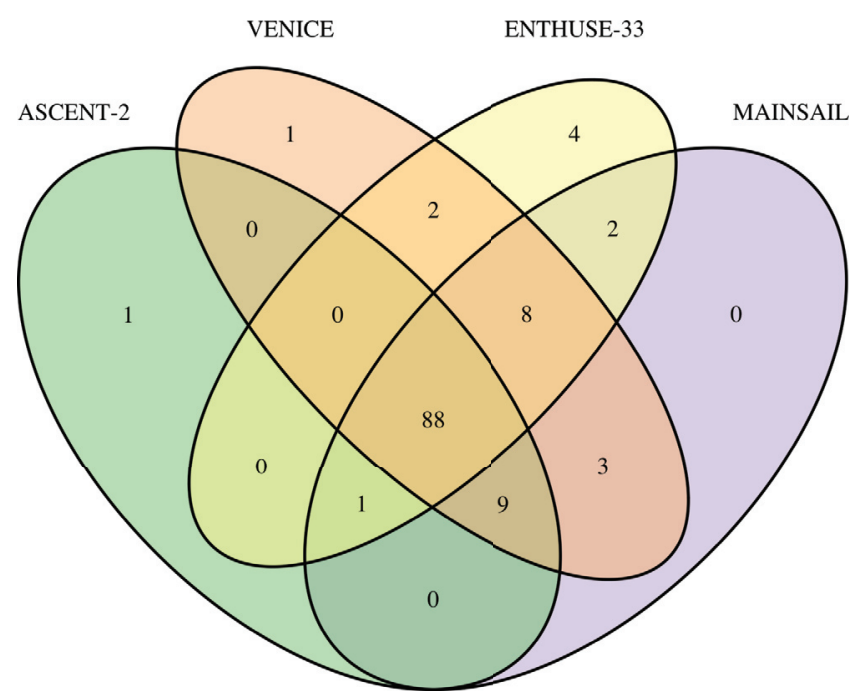

Figure 1. Variables available in the four trials ASCENT-2, MAINSAIL, VENICE, and ENTHUSE-33. 


\begin{abstract}
Table 1. Significance in univariate Cox models for variables which were not available in at least one training study.
\end{abstract}

\begin{tabular}{|c|c|c|c|c|c|}
\hline Variable & $p$-value & Significance & in ASCENT-2 & in MAINSAIL & in VENICE \\
\hline Albumin & $<0.0001$ & $\star * *$ & no & yes & yes \\
\hline Lactate dehydrogenase & $<0.0001$ & $* \star *$ & (no) & yes & yes \\
\hline Red blood cells & $<0.0001$ & $\star \star \star *$ & no & yes & no \\
\hline Sodium & $<0.001$ & $\star \star$ & no & yes & yes \\
\hline Phosphorus & $<0.05$ & . & no & yes & yes \\
\hline Region North America & $<0.05$ & . & no & yes & yes \\
\hline Region Western Europe & $<0.05$ & . & no & yes & yes \\
\hline Target lesions & $<0.05$ & . & no & yes & yes \\
\hline Region Other & 0.0651 & & no & yes & yes \\
\hline Region South America & 0.0882 & & no & yes & yes \\
\hline Non-target lesions & 0.2155 & & no & yes & yes \\
\hline Blood urea nitrogen & 0.2163 & & no & yes & yes \\
\hline Lymphocytes & 0.2223 & & no & yes & no \\
\hline Neoplasms & 0.3242 & & no & no & yes \\
\hline Total protein & 0.3832 & & no & yes & yes \\
\hline $\begin{array}{l}\text { Calculated creatinine } \\
\text { clearance }\end{array}$ & 0.5784 & & no & yes & yes \\
\hline Magnesium & 0.7174 & & no & yes & yes \\
\hline Glucose & 0.7910 & & no & yes & yes \\
\hline
\end{tabular}

subject-matter knowledge into the procedure. The minimal adaptation approach followed recommendations typically provided in statistical packages. We excluded variables with more than $10 \%$ missing values in either the training or test set, while for variables with less than $10 \%$ missing values, we used imputation, replacing the missing values with the mean value among observations that were not missing. For the second more intensive strategy, we performed subject-matter informed data cleaning, such as including additional information from the event tables, preprocessing the data, including new variables such as principal components, a toxicity score and interaction effects. The extra effort for the second approach paid off in terms of substantially increasing validation accuracy on the external test set as shown in Table 3. Details of the second approach are provided below.

High-cost data cleaning and preprocessing. An essential component for developing the final predictions for both sub-challenges 1a and $1 \mathrm{~b}$ was a comprehensive interdisciplinary exploration of the data. We built a cleaned and preprocessed dataset comprising information from the provided covariate and event tables as described in this section.

Cleaning of core table. In a first data cleaning, we identified incomplete (e. g. more than $70 \%$ missing values in either the training data or the test data), inconsistent (e. g. different levels between trials for categorical data) or irrelevant (e. g. the same value for all or almost all patients) covariables in the core table and modified the datasets accordingly: We unified categories for height, weight, race and region and removed variables with either very large fractions of missing values, redundant information or hardly any variability.

Event tables. We derived baseline patient information from the event tables as follows: The PriorMed table contained information about the medication that patients received prior to their participation in the clinical trials. Categorical assignments for medications were often missing, sometimes erroneous, and categories differed between trials. Based on our clinical expertise, we assigned appropriate categories to each medication. We then introduced new variables counting for each patient the number of medications from each category. Studies substantially differed in distributions of numbers of prior medications. We suspected that this was due to reporting biases. We hence scaled the new variables such that they had identical mean and variance across studies. The MedHistory table contained information about medical diagnoses that patients got prior to their participation in the clinical trials. For each patient, we counted the number of diagnoses in the various categories. We excluded categories which we assumed not to be clinically relevant for death or treatment discontinuation. We also deleted categories where diagnoses were reported for less than $2 \%$ of the training or test patients. From the LesionMeasure table, we extracted information such as the number of target and non-target lesions, counts per tissue and maximum target size. We noticed systematic differences 
in numbers of reported lesions between studies. We suspected that these differences were due to different reporting behaviour rather than different patient properties. In compliance with the guidelines by Eisenhauer et al. ${ }^{18}$, we only used the five largest target lesions for covariable generation and limited the number of target lesions per tissue to two. From the VitalSign table, we used patient-specific information about pulse and blood pressure. From the LabValue table, we derived covariables with additional lab test results. Difficulties were different units and truncated lab values.

Preprocessing. There were a number of values that appeared to be outliers in the statistical sense. However, though being extreme, many of these values were clinically not impossible. In order to not throw away important information, we only removed values where hemoglobin was less than five or the prostate specific antigen or platelet count were equal to zero. For ASCENT-2, there was no event data on lesions. Hence, we set the variables for the presence of target or non-target-lesions to NA ("no information") rather than NO ("no lesions found"). We log-transformed the most skewed continuous variables (prostate specific antigen, alkaline phosphatase, aspartate aminotransferase, lactate dehydrogenase and testosterone). We included selected interactions of covariables in the model, based on the results of all pairwise Cox models with two main effects and an interaction. If the coefficient of the interaction term was larger than 0.1 in its absolute value, and the p-value of the coefficient was less than 0.05 after multiple testing correction, the combination was included in the list. From the final dataset, we removed variables such that afterwards all pairwise Pearson correlations were below 0.95 in absolute value.

Several covariables were generally observed in one or several of the studies but missing for single patients. We imputed these missing values with 5 -fold multivariate imputations by chained equations (MICE) using the R package mice ${ }^{19}$ with default settings, $\mathrm{R}$ version 3.2.1. This imputation approach has proven to be successful for a variety of cancer specific data ${ }^{20-23}$.

New variables. We introduced a number of additional newly-derived variables to the set already described above: First, we aimed to represent the information from the large number of newly derived covariables from the event data tables by a smaller number. To that end, we performed a principal component analysis (PCA) once on the new variables from MedHistory and once on the new variables from LesionMeasure. We included the most important principal components as additional covariables until $95 \%$ of the variance was explained. The original variables derived from the event tables remained in the dataset as well. As a second measure, we introduced a toxicity score for each patient based on lab value information. In this variable, we combined all toxicity grades which were either provided in the LabValue table or which we derived from literature research, using databases from the U.S. Department of Health and Human Services, Food and Drug Administration (http:// www.fda.gov/downloads/BiologicsBloodVaccines/GuidanceComplianceRegulatoryInformation/Guidances/Vaccines/ucm091977. pdf), The International Clinical Studies Support Center (ICSSC, http://www.icssc.org/Documents/Resources/AEManual2003AppendicesFebruary_06_2003 final.pdf), and HSeT - Health Teaching Portal (http://hset.bio-med.ch/cms/Default.aspx?Page=12173).
Third, as the reference method by Halabi et al. ${ }^{24}$ was successful, we included their risk score as an additional covariable.

\section{Second concept: wisdom of the crowd}

Wisdom of the crowd philosophically asserts that a prediction gauged among a group of experts will be more accurate than any single prediction; the readable book by Surowiecki provides tantalizing historical and contemporary examples ${ }^{25}$. Wisdom of the crowds underpins the Sage Bionetworks DREAM challenge efforts behind crowdsourcing and citizen science, the opening of challenges to mass numbers of competitive teams on the internet or active members of the public, which has brought about improvements in breast cancer prognostic modeling among other efforts ${ }^{26,27}$. Wisdom of the crowds also underpins the accepted notion that ensembles of models confer better predictive accuracy than single models, are more robust than single methods, and have the added advantage of appropriately accounting for uncertainty ${ }^{28}$. The ability to test models on parts of the withheld test set influenced the choice of which models should be contained in the ensemble; one could term this supervised ensemble construction. We herein describe the approach.

Model averaging. In the first concept we described our multiple imputation approach for missing values. However, we noticed that distributions of variables differed between trials. Hence, we decided to only impute within the trials, not across. In other words, values were imputed based on covariable information only from patients within the same study.

Our question was then how to deal with variables that were (almost) completely missing in one entire training study but measured in other training studies. Our solution was to estimate seven different models, each taking into account a different subset of the three studies ASCENT-2, MAINSAIL and VENICE (see Figure 2). Depending on the set of studies, different covariables could be included in the model. For example, lactate dehydrogenase was completely missing in VENICE, but not in ASCENT-2 and MAINSAIL. It was hence excluded in every model based on VENICE but not otherwise. Table 2 contains two more examples. Once we had fixed the model and corresponding data, we jointly scaled the explanatory variables of all training and test studies to mean zero and variance one.

\section{Third concept: recalibration}

Recalibration of a model encompasses any manner of change to the model using data or information from the target model. In the Prostate Cancer DREAM Challenge, patient-level data from the test set did not include the target variables. Recalibration was still possible as described in the following.

High-risk and low-risk recalibration (sub-challenge 1a). With the averaged Cox model described in the previous section, we expected to predict the risks for "average patients" satisfyingly well. For high-risk or low-risk patients, however, we aimed to further improve the predictions. Hence, we adapted the scores by estimating two more models: (i) a high-risk model, where we modified the target variable DEATH (indicating whether death had been observed) such that it only counted events that happened prior to 14 months, and (ii) a low-risk model, where we only considered events that occurred after 18 months. We then recalibrated the risk scores for the 


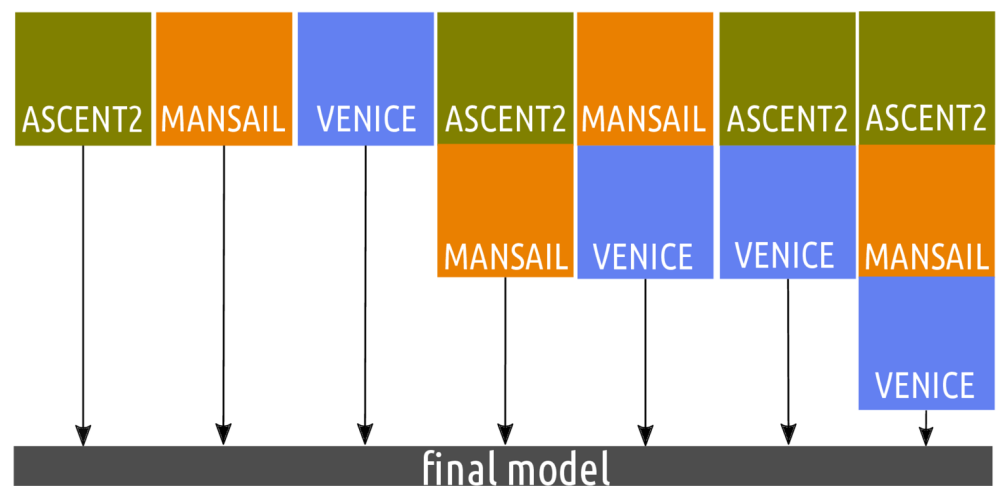

Figure 2. Datasets contained in different models to be averaged.

Table 2. Datasets contained in different models to be averaged, exemplified on selected variables.

\begin{tabular}{|c|c|c|c|c|c|c|c|}
\hline $\begin{array}{l}\text { Model name } \\
\text { Studies included }\end{array}$ & $\stackrel{1}{1}$ & $\begin{array}{c}2 \\
\text { MAINSAIL }\end{array}$ & $\begin{array}{c}3 \\
\text { VENICE }\end{array}$ & $\begin{array}{l}12 \\
\text { ASCENT-2, } \\
\text { MAINSAIL }\end{array}$ & $\begin{array}{c}13 \\
\text { ASCENT-2, } \\
\text { VENICE }\end{array}$ & $\begin{array}{c}23 \\
\text { MAINSAIL, } \\
\text { VENICE }\end{array}$ & $\begin{array}{c}123 \\
\text { ASCENT-2, } \\
\text { MAINSAIL, } \\
\text { VENICE }\end{array}$ \\
\hline $\begin{array}{l}\text { Lactate } \\
\text { dehydrogenase }\end{array}$ & yes & yes & no & yes & no & no & no \\
\hline Albumin & no & yes & yes & no & no & yes & no \\
\hline $\begin{array}{l}\text { Prostate specific } \\
\text { antigen }\end{array}$ & yes & yes & yes & yes & yes & yes & yes \\
\hline
\end{tabular}

Table 3. Comparison of prediction performance

for minimal-adaptation vs. high-cost data preprocessing.

\begin{tabular}{|l|c|c|}
\hline & $\begin{array}{c}\text { Minimal-adaptation } \\
\text { (standard) data }\end{array}$ & $\begin{array}{c}\text { High-cost } \\
\text { (improved) data }\end{array}$ \\
\hline iAUC (1a) & 0.7535 & 0.7642 \\
\hline RMSE (1b) & 304.79 & 292.15 \\
\hline
\end{tabular}

following patients: (i) For patients with risk score above the median, we calculated the average between the initial prediction and the high-risk score and considered this as the new risk score, and (ii) for those patients whose risk score was below the 25-percentile, we calculated the average between the initial model and the lowrisk model. In both cases, we made sure that the modifications only altered the ranks of patients within the defined ranges, i.e. above the median and below the 25-percentile with respect to the initial risk score. Figure 3 shows the former (x-axis) vs. the new rank (y-axis) for each patient, where a low rank means a low risk of dying.

Quantile recalibration (sub-challenge 1 b). As described above, we estimated a Cox model with lasso regularization. Based on the estimated coefficients from the training datasets, we predicted a survival curve for each of the patients in the test data. From each survival curve we derived a point estimate for the time of death as follows: A typical estimate would have been the median. However, in the training data this estimate was not optimal with respect to RMSE. We hence determined from the training data the value of $\alpha$ such that

$$
\sum_{i=1}^{n} D_{i}\left(Q_{\alpha i}-y_{i}\right)^{2}
$$

was minimized. In this formula, $n$ is the number of patients in the training data, $Q_{\alpha i}$ denotes the $\alpha \cdot 100 \%$-quantile in the survive curve for patient $i, y_{i}$ is the observed time of death for patient $i$ (can be any value if death is not observed), and $D_{i}=1$ is the indicator that death of patient $i$ has been observed (otherwise $D_{i}=0$ ). The resulting value of $\alpha$ was 0.69 . We hence derived the $69 \%$-quantiles from the survival curves as final prediction as illustrated in Figure 4.

Validation by calibration (sub-challenge 1 b). In addition to the above calibration of times to event, we also applied the validationby-calibration method by Van Houwelingen ${ }^{29}$ in sub-challenge $1 \mathrm{~b}$. This method adjusts the original predictions by rescaling them to the range of the observed outcomes using linear regression. The original method splits the training data into two subsets for model 


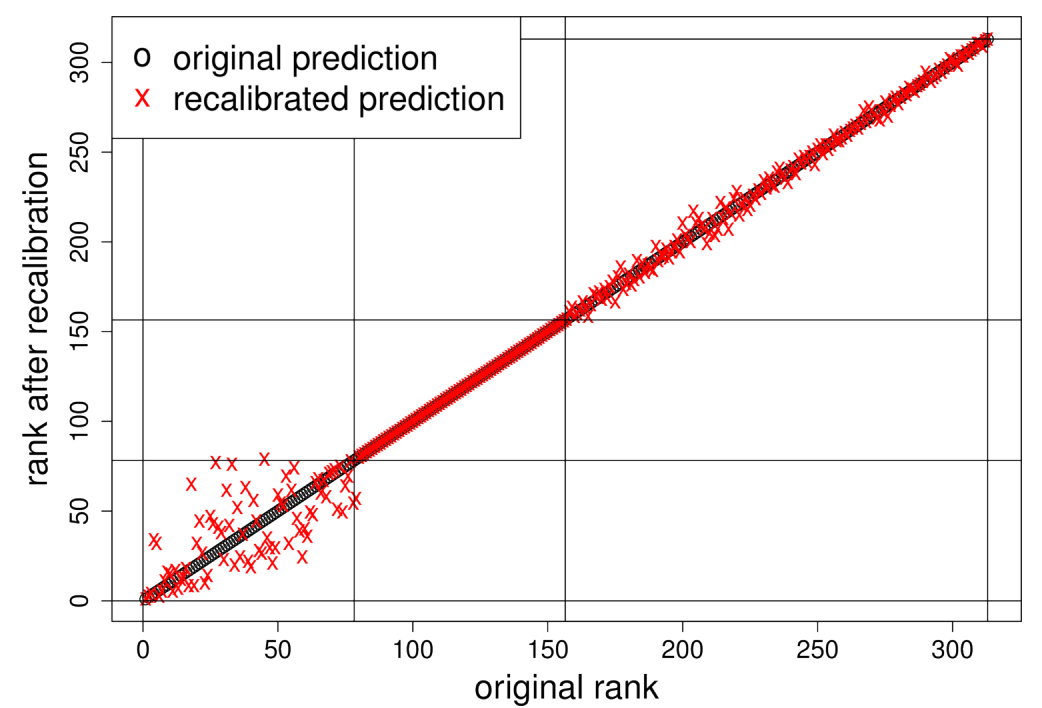

Figure 3. Recalibration for high and low risks: former (x-axis) vs. the new rank (y-axis) for each patient, where a low rank means a low risk of dying.

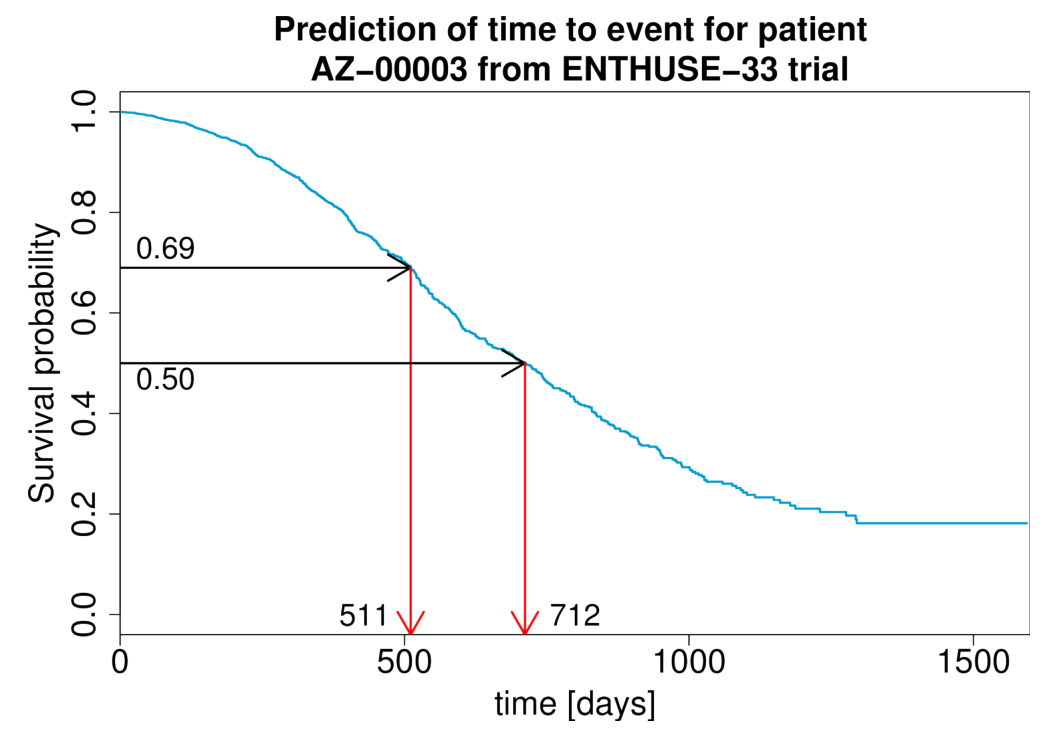

Figure 4. Time to event prediction via $69 \%$-quantile vs. $50 \%$-quantile for one selected patient. 
building and validation. For computational reasons, we omit this step. Adapted to the context here, validation-and-calibration works as follows:

1. Estimate a Cox-lasso model based on the three training trials (ASCENT-2, MAINSAIL, VENICE) as described above. From this model, compute survival curves and estimate the times to event for each patient in the training data. Let $\hat{y}$ be the predictions for those patients where death was observed, and $y$ be the corresponding observed times of death. From the same model, estimate the times to event for all patients in the test set (ENTHUSE-33) and denote them by $\hat{z}$.

2. Plot $y$ versus $\hat{y}$ and decide whether a linear relationship of the two variables can be assumed. If so, proceed.

3. Estimate a linear model $y=\beta_{0}+\beta_{1} \hat{y}+\varepsilon$ with $\varepsilon \sim N\left(0, \sigma^{2}\right)$. Let $\hat{\beta}_{0}$ and $\hat{\beta}_{1}$ be the estimated intercept and slope coefficient.

4. Recalibrate the predictions for the test patients to $\hat{z}_{c}=\hat{\beta}_{0}+\hat{\beta}_{1} \hat{z}$.

Figure 5 illustrates the procedure on our training data.

\section{Results}

We applied the three general concepts to the prediction problems of sub-challenges $1 \mathrm{a}$ and $1 \mathrm{~b}$. The benefit of applying each of the principles on the iAUC and RMSE in the
Prostate Cancer DREAM Challenge is quantified in Figure 7 and Figure 8, respectively. Details are given in the following.

\section{Impact of good data}

In order to assess the gain of the elaborate data preprocessing as compared to the low-cost minimal adaptation approach, we predicted the risk of death (sub-challenge 1a) and the time to death (sub-challenge $1 \mathrm{~b}$ ) for both data preparations. Table 3 shows the respective validation measures iAUC and RMSE when a Cox model with lasso regularization is applied as described above. For sub-challenge $1 \mathrm{~b}$, we used median survival times from the estimated survival curves. Prediction improved substantially for the high-cost data preparation with respect to both measures: The iAUC (sub-challenge 1a) increased by more than 0.01 units from 0.7535 to 0.7642 . The RMSE (sub-challenge $1 \mathrm{~b}$ ) decreased by more than 10 units from 304.79 to 292.15 .

\section{Impact of wisdom of the crowd}

We estimated the seven models described in the model averaging section above (see also Figure 2) on the training data and got a risk prediction for the test data for each of these. We then took the average of the seven predictions (each of which was again an average over five imputed datasets) to arrive at a final risk score. Compared to the standard approach (no splitting into submodels), this model averaging approach yielded improvements in terms of iAUC and RMSE measures. This is shown in Table 4 where both the standard approach model and the averaging approach employ the improved data as described in the first concept. While the increase in iAUC is again around 0.1 units from 0.7642 to 0.7733 , the improvement of the prediction of time to event is considerably

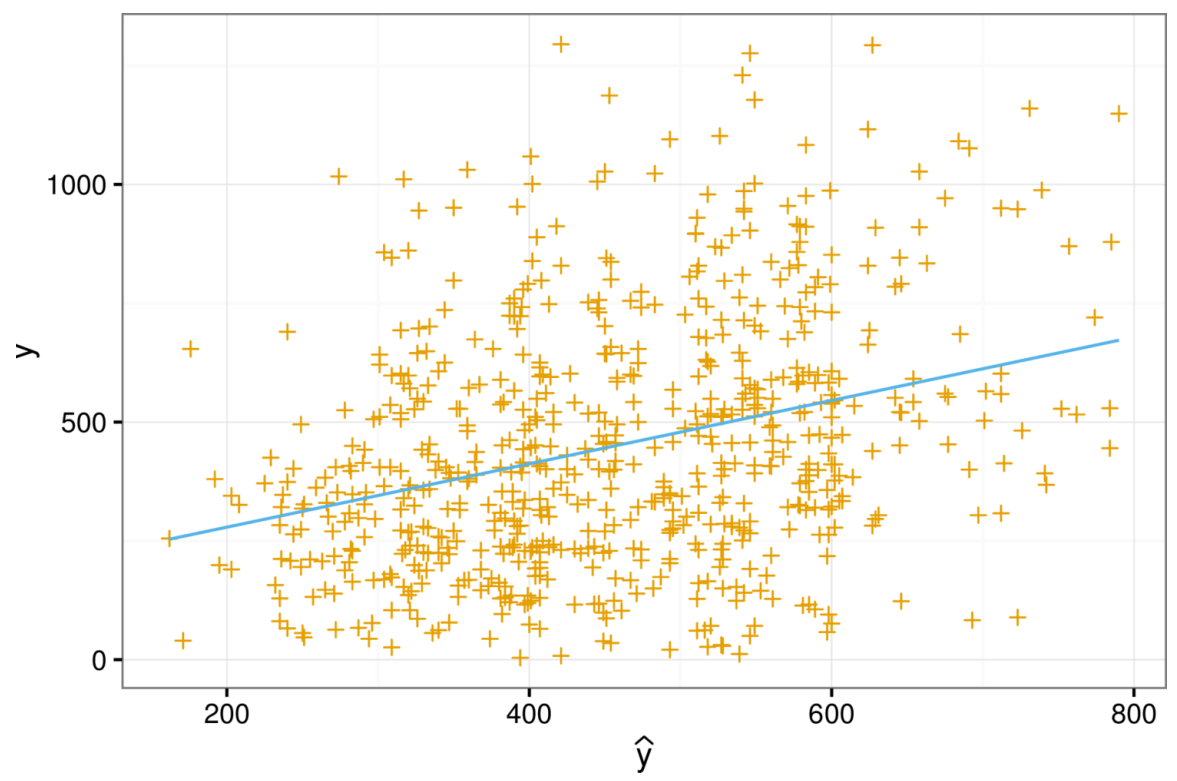

Figure 5. Linear relationship between predicted and observed times to event for the training data, used as a basis for the validation-by-calibration method. 
Table 4. Comparison of prediction performance for standard approach vs. model averaging.

\begin{tabular}{|l|c|c|}
\hline & Standard approach & Model averaging \\
\hline iAUC (1a) & 0.7642 & 0.7733 \\
\hline RMSE (1b) & 292.15 & 263.37 \\
\hline
\end{tabular}

more dramatic as it decreases the RMSE by almost 30 units from 292.15 to 263.37 .

\section{Impact of recalibration}

We applied the three proposed recalibration techniques to our predictions for risk of death (sub-challenge 1a) and time of death (sub-challenge $1 \mathrm{~b}$ ) and validated the effects of these measures on the test data (ENTHUSE-33).

Sub-challenge 1a. For the risks of dying, we once applied the low-risk calibration only, the high-risk calibration only, and both measures simultaneously. Table 5 summarizes the results. It shows that neither the low-risk nor the high-risk calibration had a substantial effect on the prediction performance in terms of iAUC: The low-risk calibration led to a small increase of iAUC by approximately 0.003 units from 0.7642 to 0.7668 . The high-risk calibration did not improve the prediction accuracy at all, although the ranks of patients changed.

Sub-challenge 1b. Recalibration of times to event caused a highly convincing improvement of prediction accuracy. Table 6 shows RMSE values for the $69 \%$-quantile recalibration only, for Van Houwelingen's validation-by-calibration approach only,
Table 5. Effect of low-risk and high-risk calibration on iAUC in sub-challenge $1 a$.

\begin{tabular}{|r|c|c|}
\hline iAUC & $\begin{array}{c}\text { w/o low-risk } \\
\text { calibration }\end{array}$ & $\begin{array}{c}\text { w/ low-risk } \\
\text { calibration }\end{array}$ \\
\hline w/o high-risk calibration & 0.7642 & 0.7668 \\
\hline w/ high-risk calibration & 0.7642 & 0.7668 \\
\hline
\end{tabular}

and for the two measures combined (i. e. first applying quantile recalibration and then the validation-by-calibration method). All recalibration approaches decreased the RMSE substantially by as much as around 100 days as compared to the non-calibrated predictions.

The choice of $\alpha=0.69$ for the $\alpha$-quantile recalibration had resulted from the training data only. As a further post-challenge analysis, we investigated whether this was also a good choice for the test dataset. Figure 6 shows RMSEs for the $\alpha$-quantile recalibration as well as the combination of quantile recalibration and validation-by-calibration for a grid of $\alpha$ values between 0.6 and 0.8 . On this grid, $\alpha=0.72$ was the optimal choice when applying the quantile recalibration only, but $\alpha=0.69$ was also reasonable. When followed by validation-by-calibration, the effect of $\alpha$ was hardly visible anymore. This makes validation-by-calibration an appealing approach for the prediction of times to event.

\section{Conclusion}

As data, computation, and statistical methods reach new horizons for the clinical risk prediction dreamers, this study reminds us of some timeless basics we should not forget: good data, wisdom of the crowds and recalibration. In this study we translated

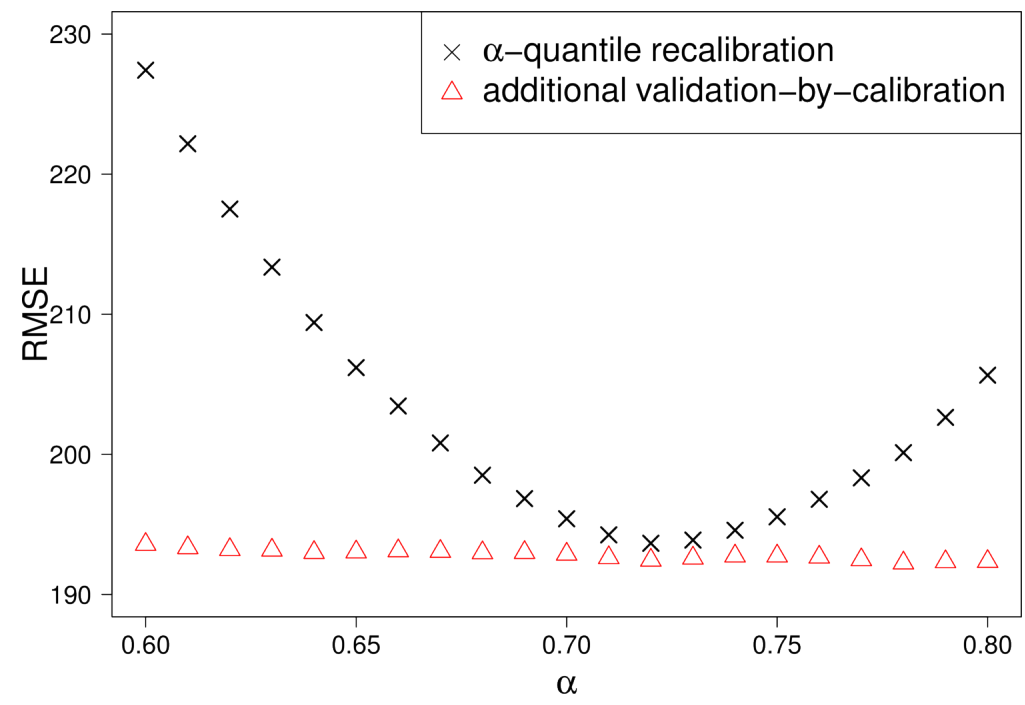

Figure 6. Effect of $\alpha$-quantile recalibration (followed by validation-by-calibration or not) on RMSE for varying $\alpha$. 
Table 6. Effect of $69 \%$-quantile recalibration and validationby-calibration on RMSE in sub-challenge $1 \mathrm{~b}$.

\begin{tabular}{|c|c|c|}
\hline & $\begin{array}{c}\text { w/o quantile } \\
\text { calibration }\end{array}$ & $\begin{array}{c}\text { w/ quantile } \\
\text { calibration }\end{array}$ \\
\hline w/o validation-by-calibration & 292.15 & 196.84 \\
\hline w/ validation-by-calibration & 194.18 & 192.99 \\
\hline
\end{tabular}

and enhanced these principles for use in developing survival risk prediction tools based on multiple heterogeneous clinical trials with large and non-overlapping sets of covariates. The impact of individual components of our proposed strategy can be quantified by their incremental influence on the assessment criteria.

The AUC is the most widely used endpoint for measuring the discrimination capability of a biomarker or risk prediction tool. However, it is limited by a lack of clinical relevance for the individual patient, defined as a comparative probability of ranks for pairs of patients, as well as a lack of statistical power being based on ranks, making it insensitive (it is invariant to monotonic changes) and notoriously difficult to budge ${ }^{30}$. Accordingly, Figure 7 shows small gains of 0.0105 points for improved data, 0.0091 additional points for model averaging, and 0.0004 additional points for recalibration, taking the best-performing option for each principle. The bottom line for implementing the three principles was to increase the iAUC from 0.7535 to 0.7768 , a minor improvement, but comparable to laudable improvements in published risk prediction tools given the robust nature of the iAUC.

The RMSE measures accuracy of a risk prediction, in other words how close a projected risk is to what actually happened to the patient, and based on the continuous measures of risk, has greater statistical power to detect differences due to technical improvements. Accordingly, more significant gains are more readily apparent in the RMSE in Figure 8, with 12.64 points for improved data, 28.78 additional points for model averaging or 99.01 additional points for recalibration, resulting in a net reduction from 111.57 points on the square root prediction scale after implementation of all three principles. Missing a large gain such as this by hastily fitting a single model without regard to the data, without averaging and without recalibration, would have cost us the challenge. But, most importantly, skipping these time-consuming basics would result in a less accurate prognosis for the individual patient.

with model averaging (wisdom of the crowd)

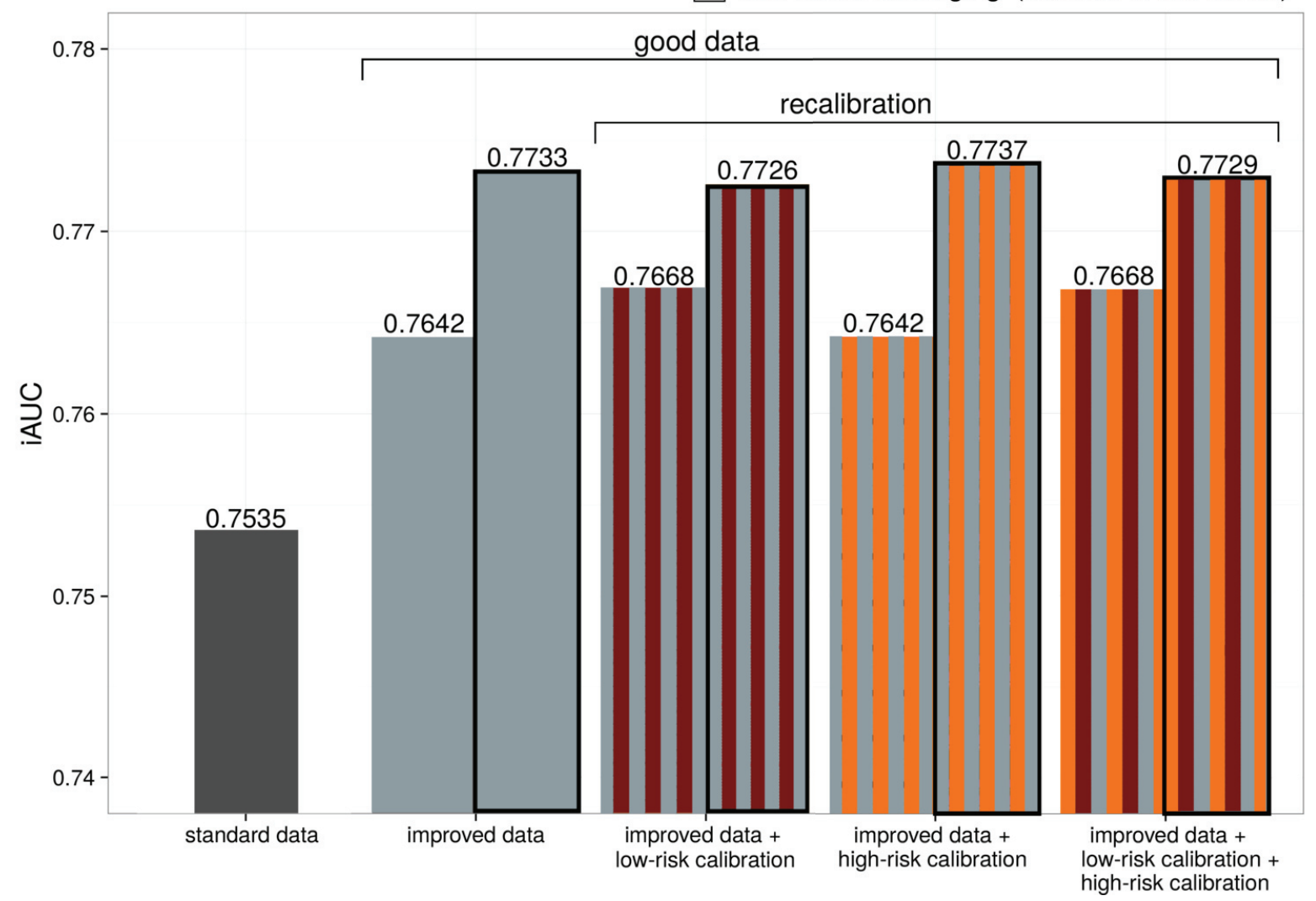

Figure 7. iAUC values resulting from different combinations of core principles to sub-challenge 1a. 
with model averaging (wisdom of the crowd)

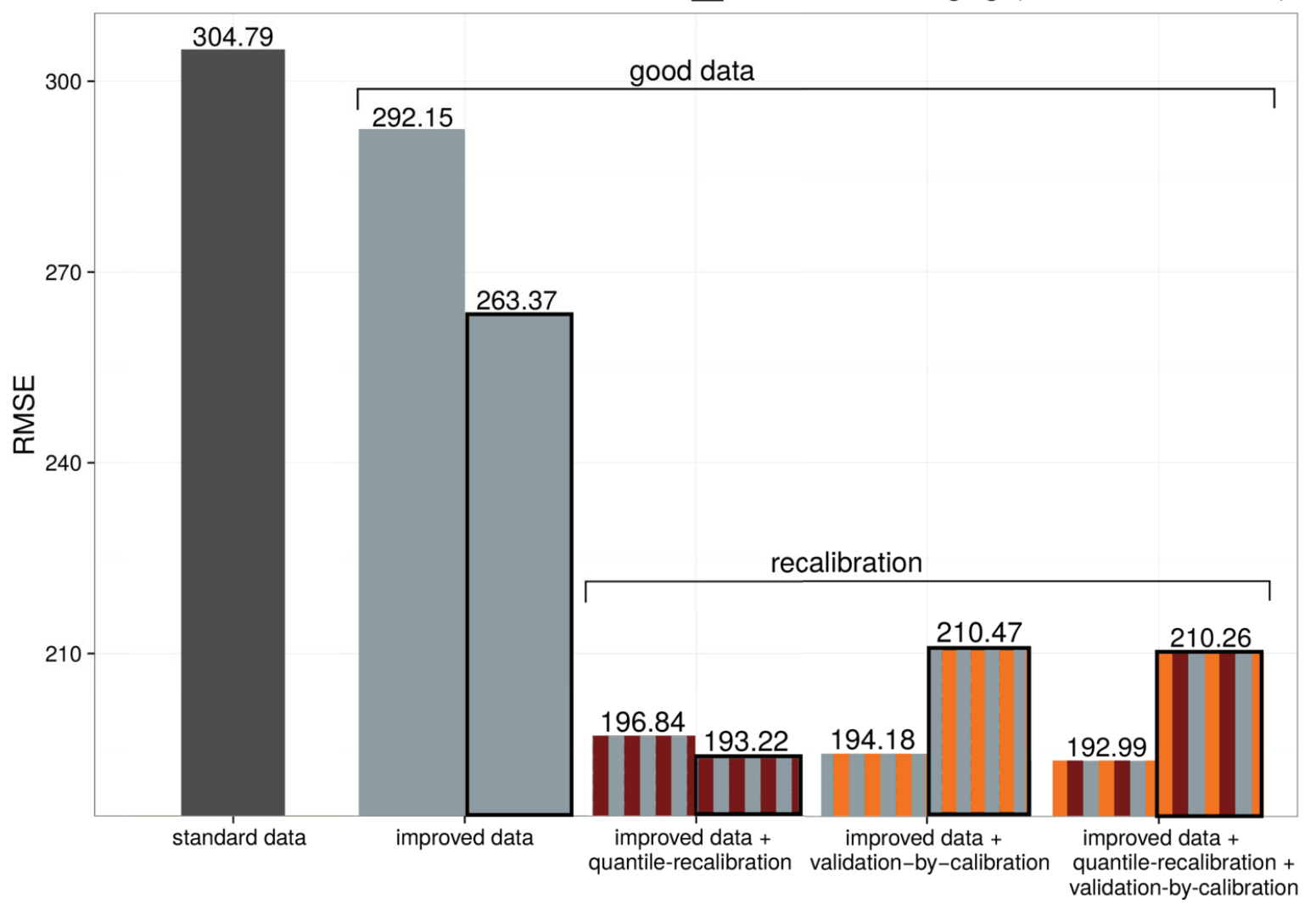

Figure 8. RMSE values resulting from different combinations of core principles to sub-challenge $1 \mathrm{~b}$.

Our data preparation included the generation of additional clinical variables. Post-challenge analyses showed that the newly introduced toxicity score was especially beneficial for good predictions in all sub-challenges, and so were the variables derived from the event data tables on lesion measures. We thus propose to generally capture such information in any clinical trials on prostate cancer. As more data become publicly available as a resource for expanding clinical risk tools, it becomes tempting to think that the art of risk prediction can be automated, eliminating the need for interdisciplinary scientists to work together. This study concludes that interdisciplinary subject-matter knowledge remains essential and that building optimal risk prediction tools remains as much an art as a process.

\section{Data availability}

The Challenge datasets can be accessed at: https://www.projectdatasphere.org/projectdatasphere/html/pcdc

Challenge documentation, including the detailed description of the Challenge design, overall results, scoring scripts, and the clinical trials data dictionary can be found at: https://www.synapse.org/ ProstateCancerChallenge

The code and documentation underlying the method presented in this paper can be found at: http://dx.doi.org/10.7303/ syn $5592405^{31}$

\section{Author contributions}

CF, IK, CK and JS preprocessed the data. CF, IK, NK, CK, ML, HS and JS established first analyses. IK, NK, CK and ML performed in-depth analysis. CF, IK and ML proposed novel modeling refinements. PD assisted the team with respect to clinical questions. DPA, CF and HS advised the team with respect to statistical questions. IK was responsible for code integration. DPA and $\mathrm{CF}$ wrote the manuscript, with contributions from IK, NK, CK, ML and JS. All members proofread the manuscript. CF supervised and guided the work. All authors contributed to discussions and decision-making processes. 


\section{Competing interests}

No competing interests were disclosed.

\section{Grant information}

$\mathrm{CF}$ and IK are supported by the European Union within the ERC grant LatentCauses. CF is supported by the German Research Foundation (DFG) within the Collaborative Research Centre 1243, Subproject A17.

The funders had no role in study design, data collection and analysis, decision to publish, or preparation of the manuscript.
Acknowledgements

This publication is based on research using information obtained from www.projectdatasphere.org, which is maintained by Project Data Sphere, LLC. Neither Project Data Sphere, LLC nor the owner(s) of any information from the web site have contributed to, approved or are in any way responsible for the contents of this publication. We are grateful to Sage Bionetworks, the DREAM organization, and Project Data Sphere for developing and supplying data for the Challenge. We thank Fabian Theis and Rolf Holle for supporting us to participate in the Challenge.
1. Koenig F, Slattery J, Groves T, et al.: Sharing clinical trial data on patient level: opportunities and challenges. Biom J. 2015; 57(1): 8-26. PubMed Abstract | Publisher Full Text | Free Full Text

2. Halabi S, Small EJ, Kantoff PW, et al.: Prognostic model for predicting survival in men with hormone-refractory metastatic prostate cancer. J Clin Oncol. 2003; 21(7): 1232-1237.

PubMed Abstract | Publisher Full Text

3. Thompson IM, Ankerst DP, Chi C, et al.: Assessing prostate cancer risk: Results from the prostate cancer prevention trial. J Natl Cancer Inst. 2006; 98(8): 529-534. PubMed Abstract | Publisher Full Text

4. Chen M, Shi L, Kelly R, et al:: Selecting a single model or combining multiple models for microarray-based classifier development?--a comparative analysis based on large and diverse datasets generated from the MAQC-II project. BMC Bioinformatics. 2011; 12(Suppl 10): S3.

PubMed Abstract | Publisher Full Text | Free Full Text

5. Hede K: Project data sphere to make cancer clinical trial data publicly available. J Natl Cancer Inst. 2013; 105(16): 1159-60.

PubMed Abstract | Publisher Full Text

6. Rozengauz DE: [Tumor of the left temporal lobe of the brain simulating an otogenic abscess]. Zh Ushn Nos Gorl Bolezn. 1965; 25(3): 83-4. PubMed Abstract

7. Guinney J, Wang T, Laajala TD, et al:: Prediction of overall survival for patients with metastatic castration-resistant prostate cancer: development of a prognostic model through a crowdsourced challenge with open clinical trial data. Lancet Oncol. 2016; published online Nov 15.

Publisher Full Text

8. Kattan MW, Hess KR, Amin MB et al: American Joint Committee on cancer acceptance criteria for inclusion of risk models for individualized prognosis in the practice of precision medicine. CA Cancer J Clin. 2016.

PubMed Abstract | Publisher Full Text

9. Tibshirani R: The lasso method for variable selection in the Cox model. Stat Med. 1997; 16(4): 385-95.

PubMed Abstract | Publisher Full Text

10. Metz CE: Basic principles of ROC analysis. Semin Nucl Med. 1978; 8(4) 283-298.

PubMed Abstract | Publisher Full Text

11. Heagerty PJ, Zheng $Y$ : Survival model predictive accuracy and ROC curves. Biometrics. 2005; 61(1): 92-105.

PubMed Abstract | Publisher Full Text

12. Hung $\mathrm{H}$, Chiang $\mathrm{CT}$ : Estimation methods for time-dependent AUC models with survival data. Can J Stat. 2010; 38(1): 8-26. Publisher Full Text

13. Blanche P, Dartigues JF, Jacqmin-Gadda H: Estimating and comparing timedependent areas under receiver operating characteristic curves for censored event times with competing risks. Stat Med. 2013; 32(30): 5381-5397. PubMed Abstract | Publisher Full Text

14. Scher HI, Jia X, Chi K, et al:: Randomized, open-label phase III trial of docetaxe plus high-dose calcitriol versus docetaxel plus prednisone for patients with castration-resistant prostate cancer. J Clin Oncol. 2011; 29(16): 2191-2198. PubMed Abstract | Publisher Full Text

15. Tannock IF, Fizazi $\mathrm{K}$, Ivanov $\mathrm{S}$, et al: Aflibercept versus placebo in combination with docetaxel and prednisone for treatment of men with metastatic castrationresistant prostate cancer (VENICE): a phase 3, double-blind randomised trial. Lancet Oncol. 2013; 14(8): 760-768.

PubMed Abstract | Publisher Full Tex
16. Petrylak DP, Vogelzang NJ, Budnik N, et al.: Docetaxel and prednisone with or without lenalidomide in chemotherapy-naive patients with metastatic castration-resistant prostate cancer (MAINSAIL): a randomised, double-blind, placebo-controlled phase 3 trial. Lancet Oncol. 2015; 16(4): 417-425. PubMed Abstract | Publisher Full Text

17. Fizazi K, Higano CS, Nelson JB, et al.: Phase III, randomized, placebo-controlled study of docetaxel in combination with zibotentan in patients with metastatic castration-resistant prostate cancer. J Clin Oncol. 2013; 31(14): 1740-1747. PubMed Abstract | Publisher Full Text

18. Eisenhauer EA, Therasse $\mathrm{P}$, Bogaerts $\mathrm{J}$, et al:: New response evaluation criteria in solid tumours: revised RECIST guideline (version 1.1). Eur J Cancer. 2009; 45(2): 228-247.

PubMed Abstract | Publisher Full Text

19. van Buuren S, Groothuis-Oudshoornl K: mice: Multivariate imputation by chained equations in R. J Stat Softw. 2011; 45(3) Publisher Full Text

20. Clark TG, Altman DG: Developing a prognostic model in the presence of missing data: an ovarian cancer case study. J Clin Epidemiol. 2003; 56(1): 28-37. PubMed Abstract | Publisher Full Text

21. Royston $P$, Parmar MK, Sylvester R: Construction and validation of a prognostic model across several studies, with an application in superficial bladder cancer. Stat Med. 2004; 23(6): 907-926.

PubMed Abstract | Publisher Full Text

22. Barosi G, Bergamaschi G, Marchetti M, et al.: JAK2 V617F mutational status predicts progression to large splenomegaly and leukemic transformation in primary myelofibrosis. Blood. 2007; 110(12): 4030-4036. PubMed Abstract | Publisher Full Text

23. Fernandes AS, Fonseca JM, Jarman IH, et al:: Evaluation of missing data imputation in longitudinal cohort studies in breast cancer survival. Int $\mathrm{J}$ Knowl Eng Soft Data Paradig. 2009; 1(3): 257 Publisher Full Text

24. Halabi S, Lin CY, Kelly WK, et al: Updated prognostic model for predicting overall survival in first-line chemotherapy for patients with metastatic castration-resistant prostate cancer. J Clin Oncol. 2014; 32(7): 671-677. PubMed Abstract | Publisher Full Text | Free Full Text

25. Surowiecki J: The wisdom of crowds: Why the many are smarter than the few and how collective wisdom shapes business, economies, societies, and nations. Doubleday. 2004.

Reference Source

26. Bain R: Citizen science and statistics: Playing a part. Significance. 2016; 13(1): 16-21. Publisher Full Text

27. McCarthy N: Prognostic models: rising to the challenge. Nat Rev Cancer. 2013 13(6): 378.

PubMed Abstract | Publisher Full Text

28. Hoeting JA, Madigan D, Raftery AE, et al:: Bayesian model averaging: A tutorial. Stat Sci. 1999; 14(4): 382-417. Publisher Full Text

29. van Houwelingen HC: Validation, calibration, revision and combination of prognostic survival models. Stat Med. 2000; 19(24): 3401-3415. PubMed Abstract | Publisher Full Text

30. Ware $\mathrm{JH}$ : The limitations of risk factors as prognostic tools. N Engl J Med. 2006 355(25): 2615-2617. PubMed Abstract | Publisher Full Text

31. Kondofersky I, Laimighofer M, Kurz C, et al:: A Bavarian Dream: Methods for Challenges 1a, 1b and 2. Synapse Storage, 2016. Publisher Full Text 


\section{Open Peer Review}

\section{Current Peer Review Status: ? ?}

\section{Version 1}

Reviewer Report 19 December 2016

https://doi.org/10.5256/f1000research.9340.r18283

(C) 2016 de Bin R. This is an open access peer review report distributed under the terms of the Creative Commons Attribution License, which permits unrestricted use, distribution, and reproduction in any medium, provided the original work is properly cited.

\section{Riccardo de Bin \\ Department of Statistics, University of Oslo, Oslo, Norway}

In this paper the authors discuss three general concepts to improve the construction of a risk prediction model, namely data pre-processing, ensemble modeling and recalibration. The idea is to take advantage of the Prostate Cancer DREAM challenge to show them applied in a case study example.

I really like the idea, because in the literature there is a clear need for papers which can provide the practitioners with useful guidelines and suggestions (see, e.g., Sauerbrei et al., 2014 ${ }^{1}$ ). I was therefore very positive about this paper, especially after the first part, in which the authors show in a clear and effective way the importance and possible implementations of data pre-processing. Unfortunately, in the following the standard drops quite abruptly, and the other two concepts, ensemble modeling and recalibration, are treated in a rather superficial and sometimes misleading way.

\section{Main concerns}

The actualization of the ensemble modeling concept, that in the paper is called "model averaging", is discussed in a single paragraph, basically giving to the reader no details about its implementation. This is particularly unsatisfying in light of its good results in improving the predictions both in terms of iAUC (discrimination) and RMSE (calibration). Actually, there is a further sentence in the Result section which says something about the implementation, but it is again very cryptic. All in all, this part left me with the feeling that a reader cannot learn much on ensemble modeling from this paper.

My strongest concerns, however, are related to the recalibration part. I agree that recalibration is important to be able to profitably use a model derived on a dataset to predict occurrences on a different dataset. Nevertheless, I am not sure that the proposed solutions are sensible.

In the first approach ("high-risk and low-risk recalibration"), two further Cox models are fitted on modifications of the dataset in which the effective sample size is reduced by considering all observations either smaller or larger than a threshold as censored. I am not sure that this 
procedure makes sense and, in any case, I cannot see how it can help under the proportional hazards assumption. Either this assumption is valid, and this methods is useless, or it is not valid, and the Cox model should not be used in the first place. I am not sure, this procedure may help in checking the validity of the proportional hazards assumption, but not for recalibration. I was therefore not surprised to see, in Figure 7, that there is no substantial improvement in using this method (assuming that the proportional hazards assumption holds). As a further note, I think that there is some confusion with the terms "average patient", "high-risk or low-risk patient" in the text.

I was also pretty surprised to see van Houwelingen (2000)'s paper ${ }^{2}$ associated with the authors' third approach ("validation by calibration"). Despite the name used, the two methods do not have much in common. The main idea of van Houwelingen (2000)'s procedure is to use data from the target population to calibrate the Cox model in order to have better predictions in the new dataset. There is no artificial split in training and validation sets, as it seems to be suggested by the authors, but two separate datasets. And, in contrast to what is stated in this paper, this division cannot be avoided, otherwise it makes no sense to apply the method. In the current problem, the authors should have used the "random subsets of data from the fourth validation trial" in their steps 2 and 3. Moreover, these two steps should have been suitably modified to work for the Cox model as in formula 3 of van Houwelingen (2000). In the context of survival analysis, indeed, the "validation by calibration" cannot be performed through a linear model.

I have not be totally able to understood the idea behind the algorithm proposed in the paper, but it seems to me that the authors are simply replicating their previous procedure "quantile recalibration", just working directly on the time axis instead of through the survival curve.

\section{Further comments}

I find quite dangerous to write "After trying several machine learning and statistical models, the combined Cox proportional hazards and lasso model was chosen as it performed optimally on the validation sets". A statistical model (or machine learning procedure) should clearly not be chosen in this way, but after rational considerations, especially when the method relies on a pretty strong assumption like the Cox model. I somehow understand that here the goal is prediction and there may not be necessary to focus on the procedure to obtain the results, but such a sentence encourage the practitioners to try whatever and pick the method which, maybe by chance, seems good on the particular case. Even here, the danger of having a model which works well only for the specific subgroups of the target population and not for the whole one (a sort of "overfitting" in a broader sense) is high.

Somehow related, it is important to note that having no-overlapping Kaplan-Meier curves is a necessary but not sufficient condition for assuming the validity of the proportional hazards assumption.

I would be curious to see how much of the improvements related to pre-processing is due to the data cleaning and how much is due to the presence of new variables. In particular, whether the new variables created with the first method (PCA) are useful at all. In contrast to the second method, in which external information is included from literature research, the first method is a simple transformation of existing data, and I do not see how it can improve the prediction. The original covariates, indeed, are kept in the model, and it seems to me that there is only two times the same information. This may even be counter- 
productive, as lasso is well know to have problems in dealing with correlated variables.

\section{Minor concerns}

When mentioning methods to deal with ties in the Cox model, references should be provided and the choice implemented in the paper (if relevant) specified.

Something about the selection of the lasso tuning parameter "s" should be included, at least by describing the procedure implemented in the paper.

The definition of discrimination reported in the paper is specific for binary classification problems. It would be better to (also) have a specific formulation for the survival analysis context (see, e.g., De Bin et al., 2014³).

Tables 4 and 5 do not provide any information in addition to that provided by Figures 7 and 8. They should be removed.

"The second criterion of calibration focused on accuracy in terms of how Idots" --> "The second criterion focused on calibration, i.e., how Idots".

The sentence "For extension to prediction of survival up until fixed time periods that accommodate censored observations" is not clear to me, it may need to be rephrased.

Variables are sometimes called "covariates", sometimes "covariables", the notation should be consistent throughout the paper.

"survive curve" --> "survival curve".

\section{References}

1. Sauerbrei W, Abrahamowicz M, Altman DG, le Cessie S, et al.: STRengthening analytical thinking for observational studies: the STRATOS initiative.Stat Med. 2014; 33 (30): 5413-32 PubMed Abstract | Publisher Full Text

2. van Houwelingen $\mathrm{H}$ : Validation, calibration, revision and combination of prognostic survival models. Statistics in Medicine. 2000; 19 (24): 3401-3415 <3401::AID-SIM554>3.0.CO;2-2">Publisher Full Text

3. De Bin R, Herold T, Boulesteix AL: Added predictive value of omics data: specific issues related to validation illustrated by two case studies.BMC Med Res Methodol. 2014; 14: 117 PubMed Abstract | Publisher Full Text

Competing Interests: No competing interests were disclosed.

I confirm that I have read this submission and believe that I have an appropriate level of expertise to confirm that it is of an acceptable scientific standard, however I have significant reservations, as outlined above.

Reviewer Report 28 November 2016 


\section{https://doi.org/10.5256/f1000research.9340.r17698}

(C) 2016 Pölsterl S. This is an open access peer review report distributed under the terms of the Creative Commons Attribution License, which permits unrestricted use, distribution, and reproduction in any medium, provided the original work is properly cited.

\section{Sebastian Pölsterl}

Institute for Cancer Research, London, UK

Kondofersky and co-authors discuss three important aspects when developing risk prediction models: good data, model averaging, and recalibration. In their paper, they evaluate the added value of each of these concepts in the context of the Prostate Cancer DREAM challenge. Their results demonstrate that data pre-processing and deriving additional variables resulted in the largest improvement, followed by combining predictions from a diverse set of models, whereas the benefit of recalibration was modest.

The paper addresses many questions researchers are facing with when building a risk prediction model from data collected from distinct clinical trials. They elegantly overcome the problem of trial-specific variables by employing an ensemble of models, where each model is specific to a particular trial (or a subset of multiple trials), which allows to leverage the full set of variables collected for a particular trial despite there being only a small set of variables that is common to all trials. Overall, the paper is well written, easy to follow, and features an extensive set of experiments.

\section{Major concerns:}

1. Preprocessing: Some aspects of preprocessing and missing value imputation are not well explained and should be addressed in a revision of the paper. It is not clear whether "highcost data cleaning and preprocessing" was used as an alternative to the "low-cost minimal adaptation approach" or in addition to it.

1. In the paragraph on the low-cost approach, the authors say that they "excluded variables with more than $10 \%$ missing values in either the training or test set", but later in the paragraph on the high-cost method, they state "we identified incomplete (e. g. more than $70 \%$ missing values in either the training data or the test data)". If the high-cost approach follows the low-cost approach there should not be any variable with $70 \%$ missing values any more.

2. The paragraph on preprocessing on page 6 mentions that highly correlated variables were removed, but is unclear whether this step occurred before or after missing value imputation using MICE. If redundant variables were removed before imputation, this could significantly increase the error of imputed values, because in contrast to model building, highly correlated variables can be very helpful for the purpose of imputation.

3. In the description of the low-cost approach, they authors mention that missing values were imputed by the mean. How were categorical variables, which have no mean, imputed? 
4. Assuming the low-cost approach was the baseline method denoted as "standard data" in figure 7, it is difficult to judge whether the improvement due the high-cost approach was due to a less biased imputation approach (MICE instead of mean) or the addition of additional variables. It would be very interesting to see what the improvement would be if the same imputation method was used and only additional derived variables would have been added.

5. Since the standard data was augmented by several derived variables, an important question is to determine which of the derived variables had the largest impact, or if it was their combined effect that resulted in the observed improvement.

\section{Model averaging:}

1. Based on the results of figures 7 and 8 , model averaging resulted in a considerable improvement in risk prediction, however not much technical details were provided. The authors should provide a more elaborate description or refer to a previously published work describing the details.

2. In particular, the authors mention that each of the 7 combinations of trials in figure 2 was imputed independently using MICE and subsequently used to construct a penalized Cox model. In this setting, two levels of averaging are necessary, first across the multiple imputed data sets, and second across the combinations of trials. The former is usually achieved by applying Rubin's rule and the latter can be achieved using several methods ranging from simple averaging to learning an additional metamodel on top of the individual models' risk scores. A description of the approach selected by the authors would be highly appreciated.

\section{Recalibration:}

1. Kondofersky and co-authors describe an additional ensemble approach were they combine a low-risk and high-risk model with an average model. It seems that the authors assumed that the proportional hazards assumption does not hold for a subset of patients and tried to address this problem by model averaging. Although this is an interesting idea, the authors unfortunately did not provide an explanation besides their empirical evaluation - why this approach might be useful. In fact, results in figure 7 show that this approach has little benefit. It would be very interesting to investigate whether this was due to the proposed recalibration approach itself or the choice of evaluation measure, which failed to measure the improvement. The authors state the using the iAUC score to measure the benefit of their methods is flawed, because it is invariant to monotone transformations. Maybe more insight could be obtained if the iAUC score is substituted for the time-dependent Brier score, which does not suffer from this problem.

2. Little details on the construction of the low- and high-risk models has been provided. The authors write that they "modified the target variable DEATH [...] such that it only counted events that happened prior to 14 months" regarding the high-risk model and that they "only considered events that occurred after 18 months" regarding the low- 
risk model. Does this mean that some patients were excluded entirely to construct the low- and high-risk models, which would lead to right and left truncation, respectively? A Cox model trained on the truncated data would thus be biased if truncation is ignored.

3. The cut-offs for the low-risk and high-risk group is based on 25-percentile and median, respectively. Are the risk groups defined based on the training data, or are new risk groups constructed each time when predicting previously unseen data? In addition, are the same risk groups used for all 7 models in the ensemble?

4. Finally, the authors sate that "modifications only altered the ranks of patients within the defined ranges". Limiting recalibration by a hard threshold means that risk scores at the limits of the respective intervals are only allowed to move into one direction, whereas risk scores located in the middle have much more freedom to move around. It would be interesting to investigate whether a soft threshold that pulls the recalibrated risk scores towards the original threshold without establishing hard boundaries would be beneficial.

\section{Minor concerns:}

1. Preliminaries (Paragraph 1):

1. The hazard ratio should be written as $\exp \left(\beta_{\mathrm{j}}\right)$, not $\exp (\beta)$, because $\beta$ has been used as a vector before.

2. It should be mentioned that the traditional Kaplan-Meier estimator is only applicable to right-censored data, not any type of censored data.

3. To check the proportional assumption it is not sufficient that estimated Kaplan-Meier do not intersect, they should be parallel to each other. In addition, other methods such as goodness-of-fit tests can be employed as well.

\section{Preliminaries (Paragraph 2):}

1. For the sake of completeness it would be good to reference methods used for accounting for ties in event times.

3. Methods (First concept: good data)

1. It is not explicitly stated which trials comprised the training data, although this information can be inferred from table 1 .

4. Methods (Table 1):

1. The table should list exact p-values, especially for the range 0.001 to 0.05

\section{Methods (Preprocessing)}

1. Were only redundant numerical features identified and removed (using Pearson correlation) or was a similar approach applied to categorical data too? If yes, which 
measure was used to assess whether they are redundant?

6. Methods (High-risk and low-risk recalibration)

1. "we calculated the average between the initial model and the low-risk model": meaning averaging the model's predictions as for the high-risk model?

7. Methods (Quantile recalibration)

1. How was weight of the lasso penalty determined?

8. Results (Impact of wisdom of the crowd)

1. Which trials were used to obtain the "standard approach"? ASCENT-2, MAINSAIL and VENICE, or only a subset of these?

\section{Grammar:}

1. Introduction (Paragraph 3): "either for statistical reasons or grounds content"

2. Methods (Cleaning of core table): "In a first data cleaning" $\rightarrow$ add word "step" at the end

3. Methods (Second concept wisdom of the crowd):

1. "the readable book by Surowiecki": no need to emphasise that books can be read

2. "the opening of challenges to mass numbers of competitive team" $\rightarrow$ massive numbers

4. Methods (Quantile recalibration): "survive curve for patient i" $\rightarrow$ survival curve

Competing Interests: No competing interests were disclosed.

I confirm that I have read this submission and believe that I have an appropriate level of expertise to confirm that it is of an acceptable scientific standard, however I have significant reservations, as outlined above. 
The benefits of publishing with F1000Research:

- Your article is published within days, with no editorial bias

- You can publish traditional articles, null/negative results, case reports, data notes and more

- The peer review process is transparent and collaborative

- Your article is indexed in PubMed after passing peer review

- Dedicated customer support at every stage

For pre-submission enquiries, contact research@f1000.com 